Check for updates

Cite this: RSC Adv., 2017, 7, 50693

Received 29th September 2017 Accepted 21st October 2017

DOI: $10.1039 / c 7 r a 10778 a$

rsc.li/rsc-advances

\title{
Visible light-induced photocatalytic degradation of gas-phase acetaldehyde with platinum/reduced titanium oxide-loaded carbon paper
}

\author{
Soonhyun Kim, (D) *a Minsun Kim, ${ }^{a} \mathrm{Ha}-$ Young Lee $^{\mathrm{b}}$ and Jong-Sung Yu (D) *b
}

\begin{abstract}
Visible light-induced degradation of gas-phase acetaldehyde $\left(\mathrm{CH}_{3} \mathrm{CHO}\right)$ is investigated using $\mathrm{O}$-deficient reduced $\mathrm{TiO}_{2-x}$-loaded carbon paper. The carbon paper is synthesized by the pyrolysis of a filter paper, and the reduced $\mathrm{TiO}_{2-x}$ is prepared by the magnesiothermic reduction of commercial anatase $\mathrm{TiO}_{2}$. The surface areas of the non-carbonized filter paper and carbon papers obtained by carbonization at 400 , 600,800 , and $1000{ }^{\circ} \mathrm{C}$ are $4.31 \mathrm{~m}^{2} \mathrm{~g}^{-1}, 13.57 \mathrm{~m}^{2} \mathrm{~g}^{-1}, 466.81 \mathrm{~m}^{2} \mathrm{~g}^{-1}, 461.60 \mathrm{~m}^{2} \mathrm{~g}^{-1}$, and $26.28 \mathrm{~m}^{2} \mathrm{~g}^{-1}$, respectively. Although the as-prepared carbon papers possess much lower specific surface areas than that of commercial activated carbon $\left(2200 \mathrm{~m}^{2} \mathrm{~g}^{-1}\right)$, the carbon papers show better adsorption capabilities. The $\mathrm{O}$-deficient reduced $\mathrm{TiO}_{2-x}$ samples exhibit strong visible-light absorption, and the reduced $\mathrm{TiO}_{2-x}$-loaded carbon papers induce rapid degradation of $\mathrm{CH}_{3} \mathrm{CHO}$ and simultaneous generation of $\mathrm{CO}_{2}$ in both closed-circulation and continuous-flow modes under visible light irradiation $(\lambda>420 \mathrm{~nm})$, attributed to the efficient adsorption of gas-phase $\mathrm{CH}_{3} \mathrm{CHO}$ by the carbon paper and the rapid and complete degradation of $\mathrm{CH}_{3} \mathrm{CHO}$ to $\mathrm{CO}_{2}$ by the active reduced $\mathrm{TiO}_{2-x}$. This strongly suggests that the reduced $\mathrm{TiO}_{2-x}$-loaded carbon paper is an efficient composite photocatalyst for the visible light-induced photocatalytic degradation of gas-phase $\mathrm{CH}_{3} \mathrm{CHO}$.
\end{abstract}

\section{Introduction}

$\mathrm{TiO}_{2}$ photocatalysis has been extensively studied in applications for air and water purification. ${ }^{\mathbf{1 - 5}}$ Photocatalytic reactions are initiated by the absorption of ultraviolet (UV) photons, which concurrently generate conduction-band (CB) electrons and valence-band (VB) holes in the $\mathrm{TiO}_{2}$ lattice. The VB holes are strong oxidants and react with surface $-\mathrm{OH}$ groups to produce $\cdot \mathrm{OH}$ radicals. The remediation power of $\mathrm{TiO}_{2}$ photocatalysts is largely attributed to the strong oxidation potential of these ${ }^{\circ} \mathrm{OH}$ radicals. However, the wide band gap of $\mathrm{TiO}_{2}$ limits the range of solar light wavelengths that can be utilized for catalysis. Several strategies have been investigated to enhance the solar light absorption of $\mathrm{TiO}_{2}$, including metal and non-metal doping, ${ }^{6-8}$ coupling of low-band gap materials, ${ }^{\mathbf{9}, 10}$ and dye sensitization. ${ }^{\mathbf{1 1 , 1 2}}$

Many researchers focus on the photooxidation of gas-phase acetaldehyde $\left(\mathrm{CH}_{3} \mathrm{CHO}\right)$, a prevalent volatile organic

\footnotetext{
${ }^{a}$ Smart Textile Convergence Research Group, Daegu Gyeongbuk Institute of Science and Technology (DGIST), Daegu, 42988, Republic of Korea. E-mail: sh2358@dgist.ac.kr; Fax: +82-53-785-3439; Tel: +82-53-785-3410

${ }^{b}$ Department Energy Science and Engineering, Daegu Gyeongbuk Institute of Science and Technology (DGIST), Daegu, 42988, Republic of Korea. E-mail: jsyu@dgist.ac. kr; Fax: +82-53-785-6409; Tel: +82-53-785-6443

$\dagger$ Electronic supplementary information (ESI) available: $\mathrm{N}_{2}$ absorption-desorption isotherms and thermal decomposition curves of synthesized materials. See DOI: 10.1039/c7ra10778a
}

compound that causes sick-house syndrome. ${ }^{\mathbf{1 3 - 1 5}}$ The complete degradation of gas-phase $\mathrm{CH}_{3} \mathrm{CHO}$ to $\mathrm{CO}_{2}$ can be achieved with $\mathrm{TiO}_{2}$ or Au-loaded $\mathrm{TiO}_{2}$ under UV irradiation. ${ }^{16,17}$ Recently, the visible light-induced photocatalytic removal of gas-phase $\mathrm{CH}_{3} \mathrm{CHO}$ was also investigated using visible light-responsive modified photocatalysts including zeolite $/ \mathrm{WO}_{3}-\mathrm{Pt}$ hybrids, ${ }^{18}$ $\mathrm{Mn}^{4+}$-doped $\mathrm{TiO}_{2}{ }^{19}$ and $\mathrm{N}$-doped $\mathrm{TiO}_{2} \cdot{ }^{20}$

For air purification, photocatalysts immobilized on support materials are usually employed. The surface area and activity of the catalyst are often reduced by immobilization. Therefore, support materials with high surface areas are preferred for photocatalyst immobilization. The composite materials generated can show synergetic effects on adsorption and photocatalysis. $^{21}$ Activated carbon is one such commonly used support material; $;^{22-24}$ it has a high surface area that can enhance adsorption and photocatalytic activity. Previously, we prepared $\mathrm{TiO}_{2}$-embedded carbon nanofibers (CNFs) and core-shellstructured $\mathrm{CNF}-\mathrm{TiO}_{2}$ nanotubes for the photocatalytic oxidation of gaseous $\mathrm{CH}_{3} \mathrm{CHO}^{25,26} \mathrm{CNF}$ mats prepared by electrospinning and subsequent carbonization did not adsorb gasphase $\mathrm{CH}_{3} \mathrm{CHO}$, but the convenient application of CNF mats suggests that mats with improved adsorption of gas-phase $\mathrm{CH}_{3} \mathrm{CHO}$ could be widely used.

Recently, O-deficient black or reduced $\mathrm{TiO}_{2-x}$ samples have attracted great attention as new photocatalysts for light-induced water splitting towards $\mathrm{H}_{2}$ production. ${ }^{27}$ The reduction of $\mathrm{TiO}_{2}$ generates many surface defects, $\mathrm{Ti}^{3+}$ species, and $\mathrm{O}$ vacancies, 
which contribute to improved light absorption. ${ }^{28,29}$ We also investigated a new approach for a defect-induced $\mathrm{TiO}_{2-x}$ solar photocatalyst for $\mathrm{H}_{2}$ production from water and $\mathrm{CO}_{2}$ reduction to fuel. ${ }^{30,31}$ However, the approach has not yet been applied to the degradation of environmental pollutants.

In this study, carbon paper was synthesized by the simple pyrolysis of a filter paper, and O-deficient reduced $\mathrm{TiO}_{2-x}$ was prepared by the magnesiothermic reduction of commercial anatase $\mathrm{TiO}_{2}$. Then, Pt-deposited reduced $\mathrm{TiO}_{2-x}(\mathrm{Pt} / \mathrm{rT})$ nanoparticles were loaded on the as-prepared carbon papers. The physicochemical properties of the Pt@reduced $\mathrm{TiO}_{2-x}$-loaded carbon paper (Pt/rT-FPC) were measured. The adsorption of gasphase $\mathrm{CH}_{3} \mathrm{CHO}$ by the filter paper carbon (FPC) or Pt/rT-FPC and the visible light-induced photocatalytic oxidation of adsorbed $\mathrm{CH}_{3} \mathrm{CHO}$ over the Pt/rT-FPC were investigated.

\section{Experimental details}

\subsection{Preparation of carbon paper from filter paper}

Carbon paper was prepared from a filter paper (Hyundai Micro Co., Ltd.). The filter paper was carbonized at 400, 600, 800, or $1000{ }^{\circ} \mathrm{C}$ for $2 \mathrm{~h}$ under continuous-flow Ar gas. The temperature was increased at the rate of $5{ }^{\circ} \mathrm{C} \mathrm{min}^{-1}$. The prepared samples are hereinafter denoted as FPC- $x$ where $x$ indicates the carbonization temperature. For comparison, two different carbon materials of CNFs prepared by a previously reported method $^{32}$ and commercial activated carbon (MSC, MSC-30, Kansai Coke and Chemicals Co., Ltd, Japan) were also used as substrates.

\subsection{Synthesis of reduced $\mathrm{TiO}_{2-x}$ and preparation of reduced $\mathrm{TiO}_{2-x}-\mathrm{FPC}$}

O-deficient reduced $\mathrm{TiO}_{2-x}$ was synthesized by a magnesiothermic reduction of commercial anatase $\mathrm{TiO}_{2}$ as previously reported. ${ }^{30,31}$ A $4 \mathrm{~g}$ sample of commercial anatase $\mathrm{TiO}_{2}$ (CT, particle size $<25 \mathrm{~nm}$, Sigma Aldrich) was mixed with the desired amount of $\mathrm{Mg}$ powder. The mixed powder was placed in a tube furnace and heated at $500{ }^{\circ} \mathrm{C}$ for $8 \mathrm{~h}$ under flowing $5 \% \mathrm{H}_{2} / \mathrm{Ar}$. After annealing, the Mg-treated sample was stirred in $2.0 \mathrm{M} \mathrm{HCl}$ solution for 1 day and then washed with distilled water and ethanol to remove $\mathrm{MgO}$ and other $\mathrm{Mg}$ impurities. The washed $\mathrm{Mg}$-free reduced $\mathrm{TiO}_{2-x}$ samples were dried at $80{ }^{\circ} \mathrm{C}$. Different reduced samples prepared with 0.5 and 0.75 molar ratios of $\mathrm{Mg}$ per molar amount of CT are denoted as rT-0.5 and rT-0.75, respectively. For comparison, a commercial photocatalyst of P25 $\mathrm{TiO}_{2}$ (P25, Aeroxide) was also used.

$\mathrm{Pt} / \mathrm{rT}$ photocatalysts were synthesized through the welldocumented photodeposition method. ${ }^{33}$ The reduced $\mathrm{TiO}_{2-x}$ photocatalyst $\left(0.5 \mathrm{~g} \mathrm{~L}^{-1}\right)$ was dispersed in $10 \mathrm{vol} \%$ methanol, and a proper amount of $x$ mole chloroplatinic acid hexahydrate solution was added dropwise to this dispersion under vigorous stirring for $30 \mathrm{~min}$. The $\mathrm{Pt}$ : photocatalyst weight ratio was $0.005: 1$. The suspension was illuminated with UV light $(300 \mathrm{~W}$ Xe arc lamp, Newport) through a $10 \mathrm{~cm}$ infrared (IR) water filter and a UV cutoff filter $(\lambda>295 \mathrm{~nm})$ for $30 \mathrm{~min}$. The suspension was filtered using $0.45 \mu \mathrm{m}$ polytetrafluoroethylene (PTFE) filters
(Millipore), washed with methanol solution, and dried at $80{ }^{\circ} \mathrm{C}$ in an oven to obtain Pt/rT. The photocatalyst was then loaded on the FPC. A $5 \mathrm{~mL}$ suspension of photocatalyst in distilled water was pipetted onto the center of each FPC specimen. After the dispersion of the suspension, the FPC was dried at $80{ }^{\circ} \mathrm{C}$ in an oven. The mass of the photocatalyst (Pt/rT-FPC or Pt/rT) in the sample holder was approximately $10 \mathrm{mg}$ for each loading.

\subsection{Characterizations}

Surface morphology images were obtained using a fieldemission scanning electron microscope (FE-SEM, Hitachi S48020, Japan). Transmission electron micrographs were obtained on a high-resolution transmission electron microscope (HR-TEM, Hitachi HF-3300, Japan). X-ray diffraction (XRD) patterns were obtained with an X-ray diffractometer (Panalytical, Empyrean, $40 \mathrm{kV}, 30 \mathrm{~mA})$ using $\mathrm{Cu} \mathrm{K} \alpha_{1}$ radiation $(\lambda=$ $0.154178 \mathrm{~nm}$ ) and a quartz monochromator. Raman spectra of the samples were analyzed by a Raman spectrophotometer (Thermo Scientific, Nicolet Almega XR) in the wavenumber range of $\sim 100$ to $4000 \mathrm{~cm}^{-1}$ using a $532 \mathrm{~nm}$ laser. The Brunauer-Emmett-Teller (BET) surface areas were determined from the $\mathrm{N}_{2}$ adsorption-desorption isotherms obtained at $77 \mathrm{~K}$ (ASAP 2020 Micromeritics). The surface compositions were measured by X-ray photoelectron spectroscopy (XPS, Thermo Scientific, Escalab 250Xi) using the $\mathrm{Mg}-\mathrm{K} \alpha$ line $(1253.6 \mathrm{eV})$ as the excitation source. The light absorption was analyzed by UVvisible diffuse reflectance spectroscopy (DRS) using a Cary series spectroscope (Cary 5000, Agilent Technologies).

\subsection{Visible light-induced gas-phase $\mathrm{CH}_{3} \mathrm{CHO}$ oxidation}

Photocatalytic oxidation experiments were performed by two different methods of closed-circulation mode and continuousflow mode. In closed-circulation mode, the photocatalytic oxidation of gaseous $\mathrm{CH}_{3} \mathrm{CHO}$ was determined in a closed circulating stainless steel reactor with a volume of $150 \mathrm{~cm}^{3}$ that could be divided into two parts (upper and lower) by a control valve, similar to the reactor in a previously reported experiment. ${ }^{25}$ The gases used were $\mathrm{CH}_{3} \mathrm{CHO}\left(300 \mathrm{ppmv} \mathrm{N}_{2}\right.$ ) as a $\mathrm{CH}_{3} \mathrm{CHO}$ standard, $\mathrm{O}_{2}$ (99.9999\%), and $\mathrm{Ar}(99.9999 \%)$ as the carrier gas. The concentrations of $\mathrm{CH}_{3} \mathrm{CHO}$ and $\mathrm{O}_{2}$ were 80 ppmv and $20 \%$, respectively. A sample of reduced $\mathrm{TiO}_{2-x}$-loaded FPC was placed in the lower reactor. Each sample weighed approximately $15 \mathrm{mg}$. The mixed gas passed through the empty upper reactor, and the concentration of $\mathrm{CH}_{3} \mathrm{CHO}$ in the exit stream was monitored until it reached a constant value. The gas was then circulated in the reactor by the pump. Next, the circulated gas was passed through the lower reactor by using the valve to make contact with the surface of the sample in the lower reactor. After adsorption equilibrium with the surface of the sample was established in the dark, the sample was illuminated with UV light (150 W Xe arc lamp, Abet Technologies). The distance between the sample and the lamp was $15 \mathrm{~cm}$, and a cut-off filter $(\lambda>295 \mathrm{~nm}$ for UV irradiation or $\lambda>420 \mathrm{~nm}$ for visible irradiation) was used. The removal of $\mathrm{CH}_{3} \mathrm{CHO}$ was monitored using a gas chromatograph (GC, HP6890, Agilent) equipped with a Porapak Q column, a flame ionization detector 
(FID), a $\mathrm{CO}_{2}$ methanizer ( $\mathrm{Ni}$ catalyst), and a gas-sampling valve. In the continuous-flow mode, the photocatalytic oxidation of gaseous $\mathrm{CH}_{3} \mathrm{CHO}$ was performed in the same $150 \mathrm{~cm}^{3}$ stainless steel reactor without division. The mixed gas was fed to the reactor at the total flow rate of $100 \mathrm{~mL} \mathrm{~min}{ }^{-1}$. The concentrations of $\mathrm{CH}_{3} \mathrm{CHO}$ and $\mathrm{O}_{2}$ were $60 \mathrm{ppmv}$ and $20 \%$, respectively. The gas residence time in the reactor was $1.5 \mathrm{~min}$. All other experimental conditions were the same as those in the closedcirculation experiments.

\section{Results and discussion}

\subsection{Characterization of FPCs}

Fig. 1 shows photographs and SEM images of the filter paper and the FPC-600. The filter paper is obviously transformed to carbon paper, as shown in Fig. 1a and c. The filter paper and the FPC-600 both contain fibers with some degree of variation in diameter. The diameters of the fibers are not strongly affected by the carbonization, but do show slight shrinkage.

Fig. $2 \mathrm{a}$ shows the XRD patterns of the filter paper, FPC-600, and FPC-800. The filter paper pattern exhibits two major peaks, a weak diffraction peak centered at $15.1^{\circ}$ and a strong diffusion diffraction peak centered at $22.5^{\circ}$, which are consistent with previously reported results. ${ }^{34}$ The FPC patterns each exhibit one broad peak centered at $23^{\circ}$, indicating amorphous carbon. ${ }^{23}$ The intensities of the diffraction peaks are increased as the temperature of carbonization is increased. Fig. $2 \mathrm{~b}$ shows the Raman spectra of the filter paper and the FPCs. The FPC spectra show two characteristic peaks centered at $1350 \mathrm{~cm}^{-1}$ and $1580 \mathrm{~cm}^{-1}$ corresponding to the $\mathrm{D}$ and $\mathrm{G}$ bands, respectively. ${ }^{35}$ These characteristic peaks become sharper and more intense with increased carbonization temperature. The D and G bands are associated with the vibrations of $\mathrm{sp}^{2}$ carbon atoms with dangling bonds; the integrated intensity ratio $\left(I_{\mathrm{D}} / I_{\mathrm{G}}\right)$ corresponds to the degree of graphitization. The $I_{\mathrm{D}} / I_{\mathrm{G}}$ ratios for

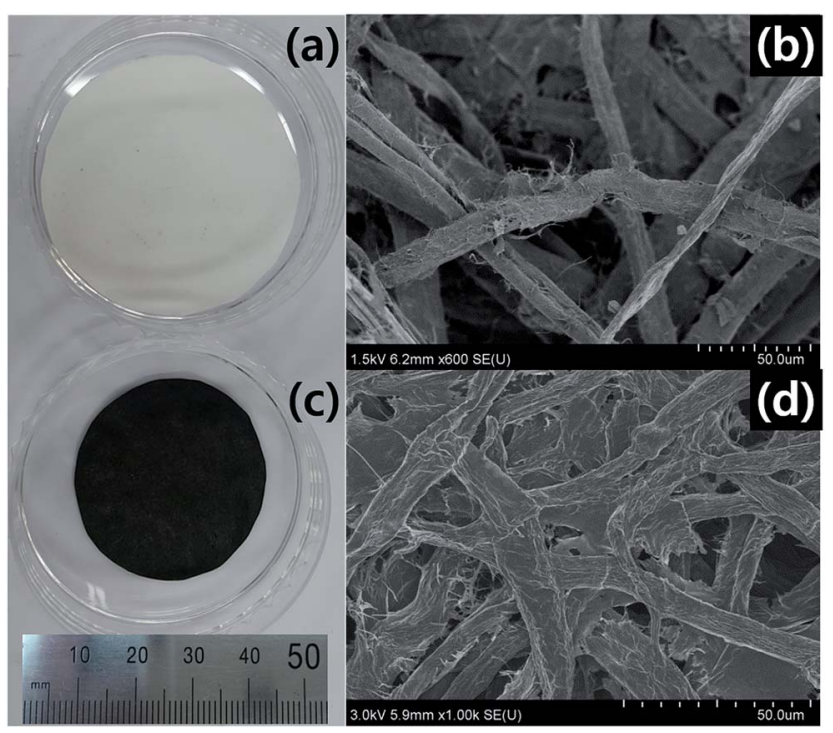

Fig. 1 Photographs and SEM images of $(a, b)$ filter paper and $(c, d)$ carbon paper after carbonization at $600{ }^{\circ} \mathrm{C}$ (FPC-600).
FPC-400, FPC-600, FPC-800, and FPC-1000 are 0.62, 0.65, 0.89, and 0.96 , respectively. This implies increasing structural order in the FPC with increasing carbonization temperature, consistent with the results reported for other cellulose materials. ${ }^{36}$ The XRD patterns and Raman spectra obviously demonstrate that graphitized carbon forms during carbonization, with increasing formation at higher carbonization temperatures.

The $\mathrm{N}_{2}$ adsorption-desorption isotherms of the filter paper and FPCs are shown in Fig. S1. $\dagger$ The BET surface areas of the filter paper, FPC-400, FPC-600, FPC-800, and FPC-1000 were $4.31 \mathrm{~m}^{2} \mathrm{~g}^{-1}, 13.57 \mathrm{~m}^{2} \mathrm{~g}^{-1}, 466.81 \mathrm{~m}^{2} \mathrm{~g}^{-1}, 461.60 \mathrm{~m}^{2} \mathrm{~g}^{-1}$, and $26.28 \mathrm{~m}^{2} \mathrm{~g}^{-1}$, respectively. The surface areas increase significantly for papers carbonized at temperatures of 400 and $800{ }^{\circ} \mathrm{C}$, and then decrease with the increase of temperature from 800 to $1000^{\circ} \mathrm{C}$. The release of volatile compounds favors the formation of pores, which create the high surface areas of FPC- 600 and FPC-800. At $1000{ }^{\circ} \mathrm{C}$, structural ordering and merging of pores decreases the surface area of the FPC.

\subsection{Gas-phase $\mathrm{CH}_{3} \mathrm{CHO}$ adsorption on FPCs}

The capability of FPCs to adsorb gaseous $\mathrm{CH}_{3} \mathrm{CHO}$ was compared with those of commercially available activated carbon (MSC), CNF, and non-carbonized filter paper. Fig. 3a shows that the dark adsorption of gaseous $\mathrm{CH}_{3} \mathrm{CHO}$ on filter paper and CNF does not occur at all, whereas MSC exhibits strong dark adsorption of $\mathrm{CH}_{3} \mathrm{CHO}$. However, FPC-800 shows better $\mathrm{CH}_{3} \mathrm{CHO}$ adsorption than MSC does. The $\mathrm{CH}_{3} \mathrm{CHO}$ adsorption may be influenced by the carbonization temperature. Therefore, the carbonization temperature-dependent adsorption of $\mathrm{CH}_{3} \mathrm{CHO}$ is analyzed as shown in Fig. $3 \mathrm{~b}$. The FPCs, except FPC-1000, exhibit strong $\mathrm{CH}_{3} \mathrm{CHO}$ adsorption. Fig. $3 \mathrm{c}$ shows the relationship between the amount of adsorbed $\mathrm{CH}_{3} \mathrm{CHO}$ and the BET surface area. The dark adsorption capacity of $\mathrm{CH}_{3} \mathrm{CHO}$ follows the order of FPC-600 > FPC-800 > FPC-400 $\gg$ MSC $\gg$ FPC-1000. Although the specific surface areas of FPCs are much lower than that of MSC, the adsorption capacities of FPCs, except that of FPC-1000, exceed that of MSC. The adsorption of $\mathrm{CH}_{3} \mathrm{CHO}$ on the surfaces of the carbon materials depends on the surface chemistry as well as the microporosity. ${ }^{37}$ The atomic ratio of $\mathrm{O}$ in the FPCs is higher than that in MSC (Fig. S3†). Therefore, enhanced adsorption of $\mathrm{CH}_{3} \mathrm{CHO}$ appears in FPCs bearing O-containing functional groups. These results were obtained in the closed-circulation system. Similar tendencies were more clearly observed in the continuous-flow system during the photocatalytic oxidation experiments. Additionally, $\mathrm{CH}_{3} \mathrm{CHO}$ showed no oxidation under UV irradiation on FPCs without photocatalyst.

\subsection{Visible light-induced activity of reduced $\mathrm{TiO}_{2-x}$}

The visible light-induced photocatalytic oxidation of gas-phase $\mathrm{CH}_{3} \mathrm{CHO}$ was further investigated using Pt/rT-FPC. Previously, reduced $\mathrm{TiO}_{2-x}$ exhibited high activity for the visible lightinduced production of $\mathrm{H}_{2}$ (ref. 30) and photocatalytic reduction of $\mathrm{CO}_{2} \cdot{ }^{31}$ In Fig. 4a, the reduced $\mathrm{TiO}_{2-x}$ samples clearly illustrate extended absorbance in the visible region as well as the UV region compared to that of commercial pristine $\mathrm{TiO}_{2}$, 

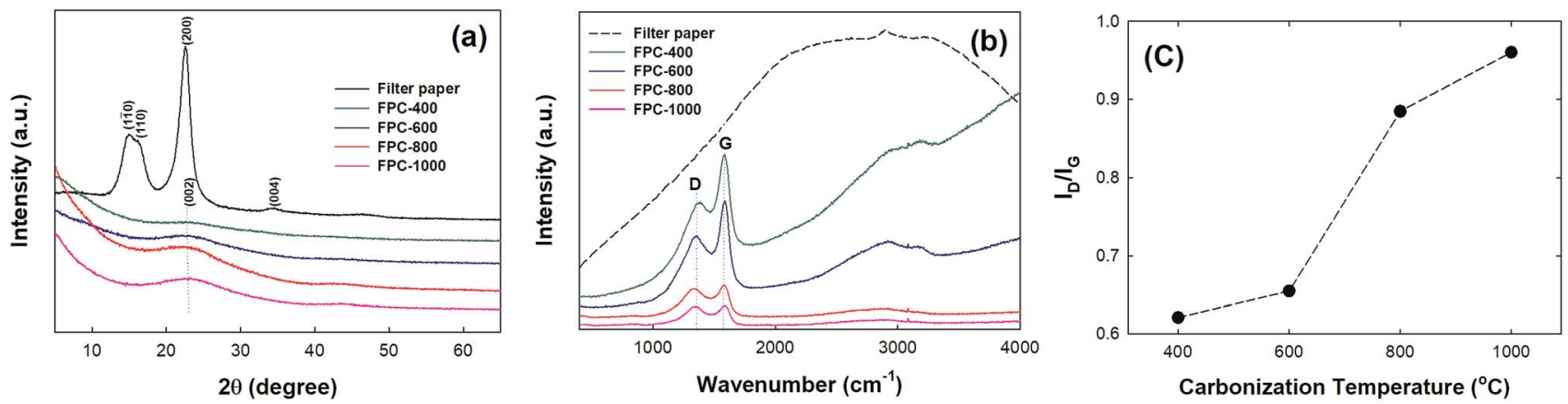

Fig. 2 (a) XRD patterns and (b) Raman spectra of filter paper and FPCs. (c) Ratio of intensity of D to G peaks in Raman spectra for carbon papers as a function of carbonization temperature.

which shows only UV-region absorption. Our previous work revealed that visible light absorption is related to the presence of $\mathrm{Ti}^{3+} \cdot{ }^{31}$ The absorption of light increases as the amount of $\mathrm{Mg}$ used in the reduction increases, as observed for rT-0.5 and rT0.75 ; this is also reflected in the color change of the samples from white to yellow and gray for pristine $\mathrm{TiO}_{2}$, rT-0.5, and rT0.75 , respectively. The XRD patterns of the different samples are presented in Fig. 4b. Despite the color changes, the reduced samples still show predominantly anatase-phase $\mathrm{TiO}_{2}$. Although the XRD analysis indicates no major structural changes during $\mathrm{Mg}$ reduction, the $\mathrm{Mg}$ treatment probably induces modifications on the surfaces of the reduced $\mathrm{TiO}_{2-x}$ particles, as reflected in the enhanced light absorption and color changes of the reduced samples. The magnesiothermic reduction of $\mathrm{TiO}_{2}$ follows the reaction shown below. The strong reduction powder of $\mathrm{Mg}$ causes the pristine $\mathrm{TiO}_{2}$ to lose the framework of $\mathrm{O}$ on the surface of the $\mathrm{TiO}_{2}$ particles, generating reduced O-deficient $\mathrm{TiO}_{2-x}$ and $\mathrm{MgO}$. The light absorption in the visible region is increased, and the color darkens because of the increased surface modification of the reduced samples with increased amounts of $\mathrm{Mg}$. In the given reaction conditions, this modification occurs only on the surface of the reduced sample;
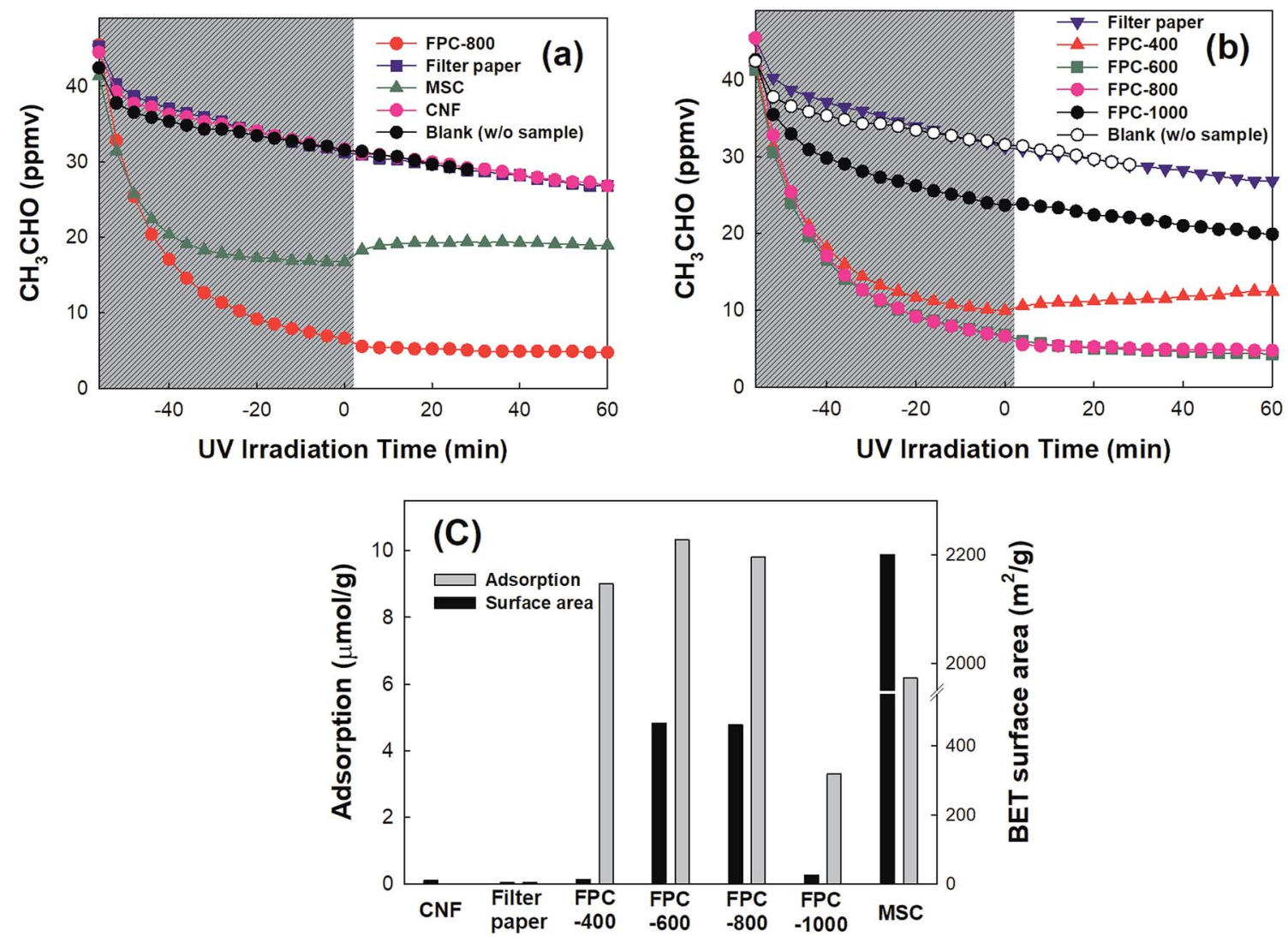

Fig. 3 (a, b) Time-profile $\mathrm{CH}_{3} \mathrm{CHO}$ concentration on the FPC before and after UV irradiation in closed-circulation mode. (c) The relationship between the $\mathrm{CH}_{3} \mathrm{CHO}$ adsorption capacity and BET surface area of the FPC. 

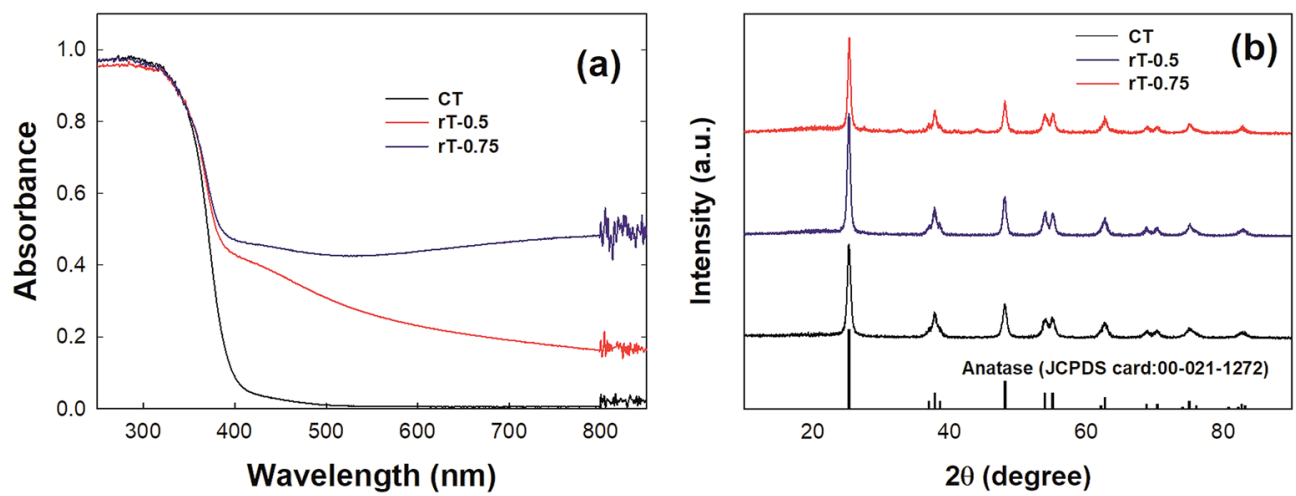

Fig. 4 (a) DRS absorbance and (b) XRD patterns of CT, rT-0.5, and $\mathrm{rT}-0.75$.

thus, the inner part of the reduced sample has the anatase $\mathrm{TiO}_{2}$ structure, as indicted by the XRD patterns in Fig. $4 \mathrm{~b}$.

$$
\mathrm{TiO}_{2}+\mathrm{Mg} \rightarrow \mathrm{TiO}_{2-x}+\mathrm{MgO}
$$

The photocatalytic oxidation of $\mathrm{CH}_{3} \mathrm{CHO}$ by reduced $\mathrm{TiO}_{2-x}$ under visible light was performed in continuous-flow mode. Fig. 5a shows the photocatalytic oxidation of $\mathrm{CH}_{3} \mathrm{CHO}$ on reduced $\mathrm{TiO}_{2-x}$ and $\mathrm{P} 25 \mathrm{TiO}_{2}$. The dark adsorption capacities on the surfaces of the reduced $\mathrm{TiO}_{2-x}$ and $\mathrm{P} 25 \mathrm{TiO}_{2}$ are much lower than those of reduced $\mathrm{TiO}_{2-x}$ immobilized on the FPCs. Under visible irradiation, the concentration of $\mathrm{CH}_{3} \mathrm{CHO}$ is only
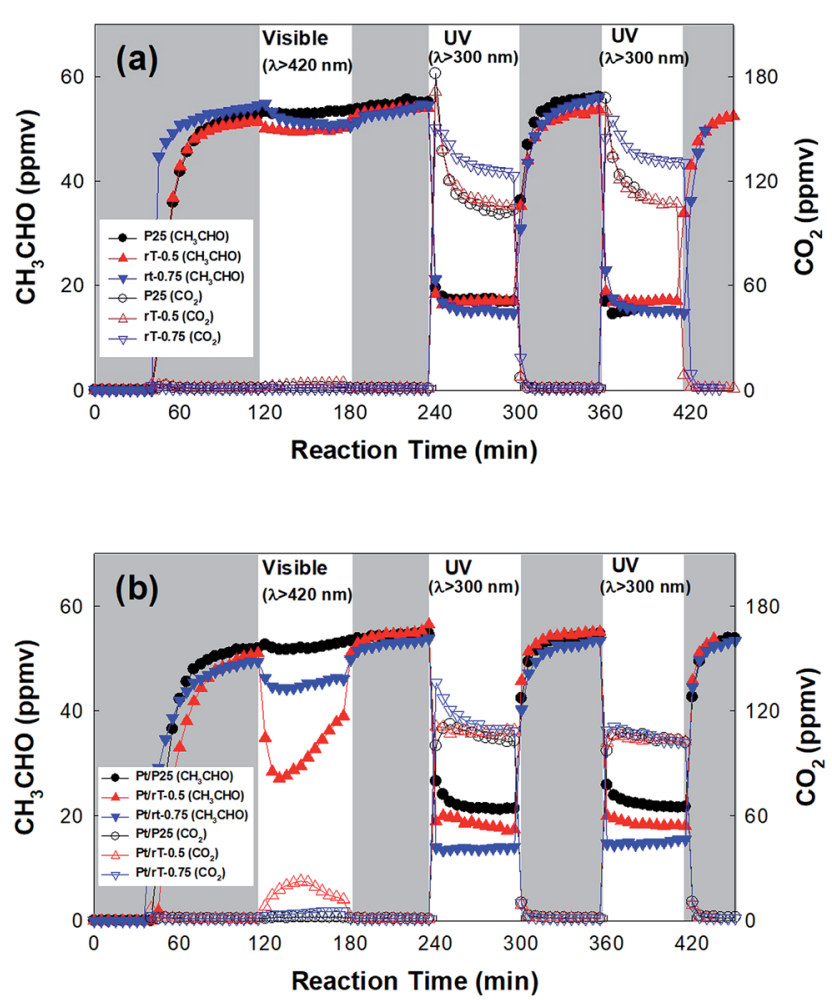

Fig. 5 Dark adsorption and photocatalytic oxidation of $\mathrm{CH}_{3} \mathrm{CHO}$ under visible and UV irradiation on (a) bare photocatalyst and (b) Ptdeposited photocatalyst in continuous-flow mode. slightly reduced and $\mathrm{CO}_{2}$ production is negligible. Under $\mathrm{UV}$ irradiation, however, the $\mathrm{CH}_{3} \mathrm{CHO}$ is degraded and $\mathrm{CO}_{2}$ immediately produced. The concentrations of $\mathrm{CH}_{3} \mathrm{CHO}$ and $\mathrm{CO}_{2}$ reach steady-state levels after several minutes, indicating that establishing adsorption-desorption equilibrium under UV illumination requires a long time. Meanwhile, the concentration of $\mathrm{CO}_{2}$ on rT-0.75 is higher than that on either P25 or rT-0.5. This implies that the photocatalytic oxidation of $\mathrm{CH}_{3} \mathrm{CHO}$ is faster on rT-0.75. The $\mathrm{CH}_{3} \mathrm{CHO}$ degradation and the $\mathrm{CO}_{2}$ production under $\mathrm{UV}$ irradiation remain almost identical throughout repeated cycling. However, although the reduced $\mathrm{TiO}_{2-x}$ absorbs visible light, the visible light-induced photocatalytic oxidation of $\mathrm{CH}_{3} \mathrm{CHO}$ occurs on neither rT-0.5 nor rT- 0.75 . This can be attributed to the rapid recombination of the electron-hole pairs produced under visible irradiation. Therefore, Pt must be photodeposited onto the reduced $\mathrm{TiO}_{2-x}$ surface for the efficient separation of the photogenerated electron-hole pairs.

Fig. 6 shows HR-TEM images of Pt/P25, Pt/rT-0.5, and Pt/rT0.75. In all cases, $\mathrm{Pt}$ is deposited similarly. The photocatalytic oxidation of $\mathrm{CH}_{3} \mathrm{CHO}$ on Pt-deposited $\mathrm{P} 25$ and reduced $\mathrm{TiO}_{2-x}$ was also investigated. As shown in Fig. 5b, under visible irradiation, the $\mathrm{CH}_{3} \mathrm{CHO}$ is degraded with simultaneous $\mathrm{CO}_{2}$ production on Pt/rT-0.5. The concentrations of $\mathrm{CH}_{3} \mathrm{CHO}$ and $\mathrm{CO}_{2}$ do not reach a steady-state level because of the offsets of the adsorption and the photocatalytic oxidation of $\mathrm{CH}_{3} \mathrm{CHO}$. This implies that Pt deposition inhibits the recombination of charge carriers created by absorbing visible light and thereby enables visible lightinduced $\mathrm{CH}_{3} \mathrm{CHO}$ degradation. The photocatalytic reaction of $\mathrm{CH}_{3} \mathrm{CHO}$ oxidation into $\mathrm{CO}_{2}$ can be written as follows:

$$
\begin{gathered}
\mathrm{Pt} / \mathrm{TiO}_{2-x}+h \nu \rightarrow \mathrm{h}^{+}\left(\mathrm{Pt} / \mathrm{TiO}_{2-x}\right)+\mathrm{e}^{-}\left(\mathrm{Pt} / \mathrm{TiO}_{2-x}\right) \\
\mathrm{CH}_{3} \mathrm{CHO}+10 \mathrm{~h}^{+}\left(\mathrm{Pt} / \mathrm{TiO}_{2-x}\right)+3 \mathrm{H}_{2} \mathrm{O} \rightarrow 2 \mathrm{CO}_{2}+10 \mathrm{H}^{+} \\
2.5 \mathrm{O}_{2}+10 \mathrm{e}^{-}\left(\mathrm{Pt} / \mathrm{TiO}_{2-x}\right)+10 \mathrm{H}^{+} \rightarrow 5 \mathrm{H}_{2} \mathrm{O} \\
\text { (Total) } \mathrm{CH}_{3} \mathrm{CHO}+2.5 \mathrm{O}_{2} \rightarrow 2 \mathrm{CO}_{2}+2 \mathrm{H}_{2} \mathrm{O}
\end{gathered}
$$

On Pt/rT-0.75, the $\mathrm{CH}_{3} \mathrm{CHO}$ is degraded but no $\mathrm{CO}_{2}$ is observed, which could be attributed to the production of some undetected intermediates by the visible light-induced 


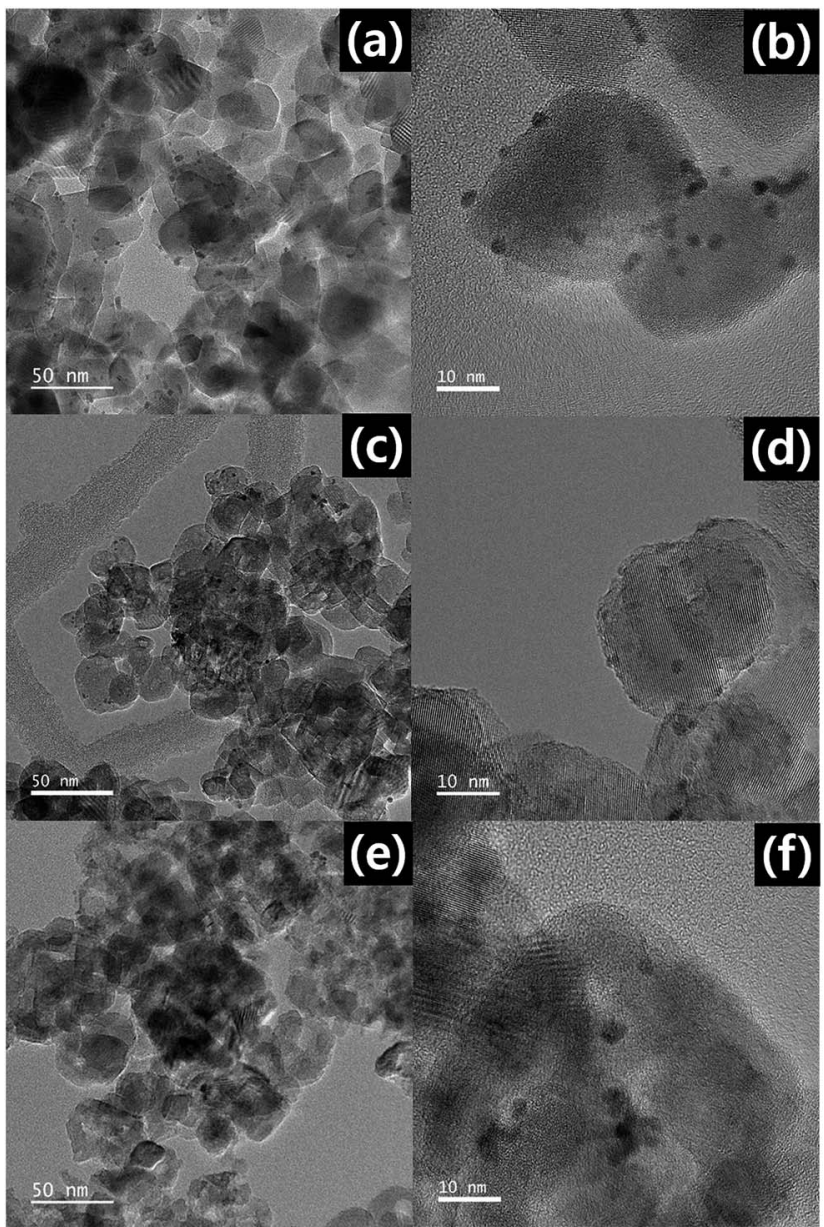

Fig. 6 High-resolution TEM images of (a, b) Pt/P25, (c, d) Pt/rT-0.5, and $(e, f) P t / r T-0.75$

degradation of $\mathrm{CH}_{3} \mathrm{CHO}$. Meanwhile on $\mathrm{Pt} / \mathrm{P} 25, \mathrm{CH}_{3} \mathrm{CHO}$ is not degraded under visible irradiation. The photocatalytic degradation rates of $\mathrm{CH}_{3} \mathrm{CHO}$ on Pt/P25, Pt/rT-0.5, and Pt/rT-0.75 under UV irradiation are much higher than those under visible irradiation. Compared to the results obtained in the absence of Pt, the photocatalytic activities for $\mathrm{CH}_{3} \mathrm{CHO}$ degradation under UV irradiation are not significantly enhanced. This implies that UV light-induced charge separation occurs efficiently on P25, rT-0.5, and rT-0.75, so the effect of Pt

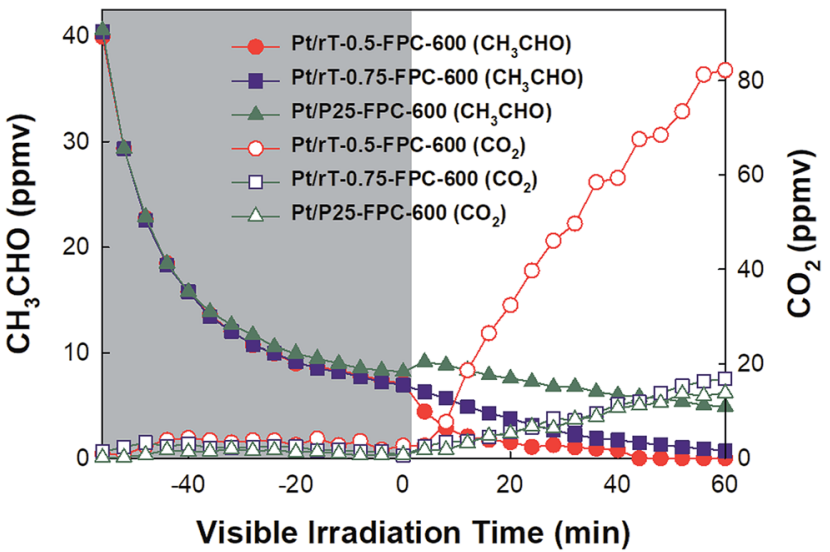

Fig. 8 Dark adsorption and photocatalytic oxidation of $\mathrm{CH}_{3} \mathrm{CHO}$ on the Pt/P25-FPC-600, Pt/rT-0.5-FPC-600, and Pt/rT-0.75-FPC-600 under visible irradiation in closed-circulation mode.

deposition is not significant. Moreover, the $\mathrm{CH}_{3} \mathrm{CHO}$ degradation and the $\mathrm{CO}_{2}$ production under UV irradiation remain almost identical throughout repeated cycling, as with the results obtained in the absence of Pt.

\subsection{Visible light-induced gas-phase $\mathrm{CH}_{3} \mathrm{CHO}$ oxidation}

The visible light-induced photocatalytic oxidation of gas-phase $\mathrm{CH}_{3} \mathrm{CHO}$ using reduced $\mathrm{TiO}_{2-x}$-loaded FPC was investigated. Based on the above results, we investigated visible light-induced gas-phase $\mathrm{CH}_{3} \mathrm{CHO}$ oxidation using the Pt-deposited photocatalysts and FPC-600. We observed cross-sectional SEM images and analyzed the elemental composition of the Pt/rT-0.5 loaded on the FPC-600 by energy-dispersive X-ray spectroscopy (EDS) mapping, as shown in Fig. $7 \mathrm{a}$ and $8 \mathrm{~b}$. The Pt/tT-0.5 is well loaded and easily distinguishable on the FPC-600. The amount of Pt/tT-0.5 nanoparticles on the surface of the FPC- 600 is indirectly calculated from the thermogravimetric analysis (TGA) results (Fig. S2 $\dagger$ ). Fig. S2a † shows that the decomposition of Pt/ rT-0.5 loaded on FPC-600 begins at approximately $400{ }^{\circ} \mathrm{C}$ and stops at $500{ }^{\circ} \mathrm{C}$. The weight loss of Pt/rT-0.5 loaded on the FPC600 is approximately $47 \%$.

Fig. 8 shows the photocatalytic oxidation of $\mathrm{CH}_{3} \mathrm{CHO}$ on Pt/ rT-0.5, Pt/rT-0.75, and Pt/P25 loaded on FPC-600 in closedcirculation mode. All cases show strong dark adsorption of

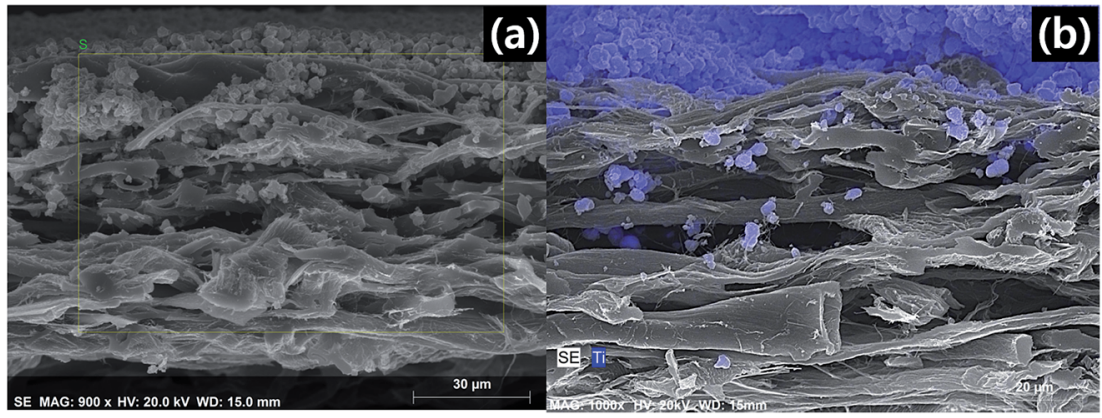

Fig. 7 Cross-sectional SEM images of Pt/rT-0.5 loaded on FPC-600. 


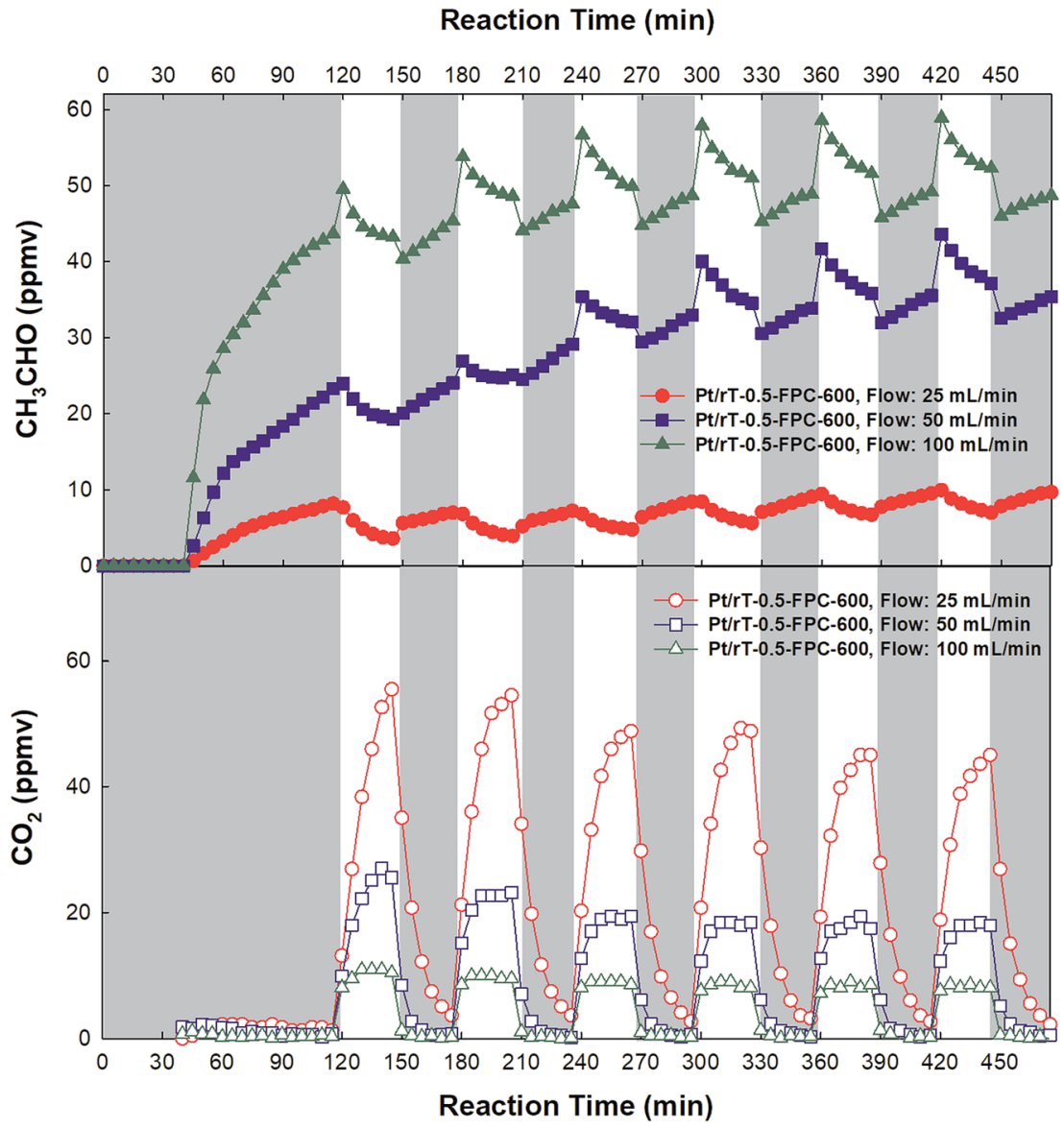

Fig. 9 Dark adsorption and photocatalytic oxidation of $\mathrm{CH}_{3} \mathrm{CHO}$ on the Pt/rT-0.5-FPC-600 under visible irradiation in continuous-flow mode.

$\mathrm{CH}_{3} \mathrm{CHO}$, similar to the dark adsorption of $\mathrm{CH}_{3} \mathrm{CHO}$ on the FPC without Pt/rT-0.5, Pt/rT-0.75, or Pt/P25. Upon visible irradiation, the $\mathrm{Pt} / \mathrm{rT}-0.5$ loaded on the FPC-600 shows rapid degradation of $\mathrm{CH}_{3} \mathrm{CHO}$ and simultaneous generation of $\mathrm{CO}_{2}$. The photocatalytic oxidation of $\mathrm{CH}_{3} \mathrm{CHO}$ on Pt/rT-0.5-FPC-600 is also performed in continuous-flow mode under repeated experiments, as shown in Fig. 9. The photocatalytic oxidation of $\mathrm{CH}_{3} \mathrm{CHO}$ to $\mathrm{CO}_{2}$ is strongly dependent on the flow rates of 25,50 , and 100 $\mathrm{mL} \mathrm{min}^{-1}$, which relate directly to the residence times of 6,3 , and $1.5 \mathrm{~min}$, respectively. For all cases, the concentration of $\mathrm{CH}_{3} \mathrm{CHO}$ does not reach steady-state levels within $30 \mathrm{~min}$. At the highest flow rate, $\mathrm{CO}_{2}$ production is rapidly saturated and its concentration remains constant under repeated experiments. However, at the lowest flow rate, the concentration of $\mathrm{CO}_{2}$ does not reach a steady-state level but $\mathrm{CO}_{2}$ production from the photocatalytic oxidation of $\mathrm{CH}_{3} \mathrm{CHO}$ is dramatic, possibly because of the longer residence time. The photocatalytic oxidation of $\mathrm{CH}_{3} \mathrm{CHO}$ to $\mathrm{CO}_{2}$ was repeated up to six times. The result demonstrates the good stability of Pt/rT-0.5-FPC-600. Investigations to improve the operation conditions are needed for photocatalytic oxidation applications. Even with a small amount of $\mathrm{Pt} / \mathrm{rT}-0.5$, the photocatalytic oxidation of $\mathrm{CH}_{3} \mathrm{CHO}$ under visible irradiation occurs in a few minutes. The FPC-600 efficiently adsorbs the $\mathrm{CH}_{3} \mathrm{CHO}$, and Pt/rT-0.5 rapidly and completely degrades the $\mathrm{CH}_{3} \mathrm{CHO}$ to $\mathrm{CO}_{2}$, even under visible irradiation. It is also possible that some electrons are transferred to the carbon paper, which would promote electron-hole pair separation; this implies that the carbon paper could function as an electron-transfer mediator as well as a support material. ${ }^{32,38} \mathrm{~A}$ schematic of the visible lightinduced photocatalytic degradation of gas-phase $\mathrm{CH}_{3} \mathrm{CHO}$ using reduced $\mathrm{TiO}_{2-x}$-loaded FPC is shown in Fig. 10.
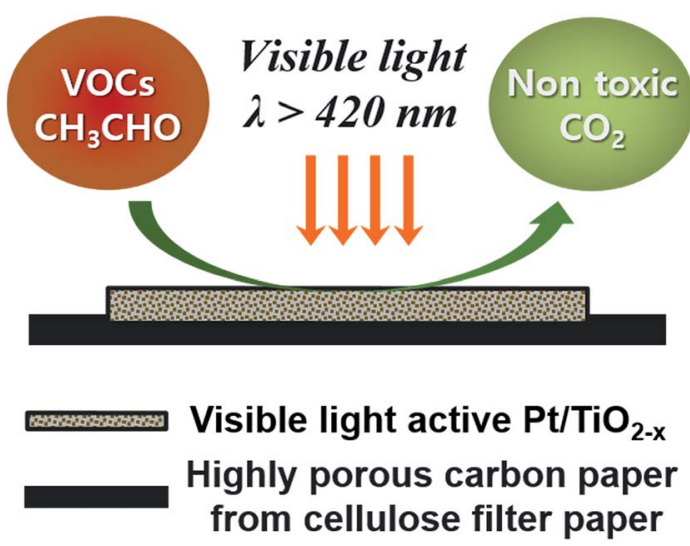

Fig. 10 Schematic of the visible light-induced photocatalytic degradation of gas-phase $\mathrm{CH}_{3} \mathrm{CHO}$ using reduced $\mathrm{TiO}_{2-x}$-loaded carbon paper. 


\section{Conclusion}

In this work, visible light-induced gas-phase $\mathrm{CH}_{3} \mathrm{CHO}$ degradation on $\mathrm{Pt} /$ reduced $\mathrm{TiO}_{2-x}$-loaded FPC was examined for the first time. The FPC was synthesized by the simple pyrolysis of filter paper; its surface area was strongly affected by the carbonization temperature. Although the specific surface areas of the FPCs were much lower than that of commercial MSC, the adsorption rate and capacities of FPCs outperformed those of MSC. The O-deficient reduced $\mathrm{TiO}_{2-x}$ prepared by the $\mathrm{Mg}-\mathrm{H}_{2}$ reduction of commercial anatase $\mathrm{TiO}_{2}$ showed increased visible light absorption; it was successfully utilized as the active material for visible light-induced photocatalysis. The reduced $\mathrm{TiO}_{2}$-loaded FPC showed rapid degradation of $\mathrm{CH}_{3} \mathrm{CHO}$ and simultaneous generation of $\mathrm{CO}_{2}$ in both closed-circulation and continuous-flow modes under visible irradiation, clearly demonstrating that the reduced $\mathrm{TiO}_{2-x}$-loaded FPC described in this work is a suitable candidate for the visible light-induced photocatalytic degradation of gas-phase $\mathrm{CH}_{3} \mathrm{CHO}$.

\section{Conflicts of interest}

There are no conflicts of interest to declare.

\section{Acknowledgements}

This work was supported by the DGIST R\&D Program of the Ministry of Science, ICT \& Future Planning (17-NT-02 and 17-01HRLA-01). This work was also supported by Basic Science Research Program, through the National Research Foundation of Korea (NRF), funded by the Ministry of Science, ICT \& Future Planning (2017R1A2B4003919).

\section{References}

1 M. R. Hoffmann, S. T. Martin, W. Choi and D. W. Bahnemann, Chem. Rev., 1995, 95, 69-96.

2 W. Choi, J. Y. Ko, H. Park and J. S. Chung, Appl. Catal., B, 2001, 31, 209-220.

3 S. Kim and W. Choi, Environ. Sci. Technol., 2002, 36, 2019-2025. 4 J. S. Park and W. Choi, Chem. Lett., 2005, 34, 1630-1631.

5 J. Ryu and W. Choi, Environ. Sci. Technol., 2008, 42, 294-300. 6 S. Kim, S.-J. Hwang and W. Choi, J. Phys. Chem. B, 2005, 109, 24260-24267.

7 J. W. Zheng, A. Bhattcahrayya, P. Wu, Z. Chen, J. Highfield, Z. Dong and R. Xu, J. Phys. Chem. C, 2010, 114, 7063-7069.

8 S. Na Phattalung, S. Limpijumnong and J. Yu, Appl. Catal., B, 2017, 200, 1-9.

9 M. Kim, Y. K. Kim, S. K. Lim, S. Kim and S.-I. In, Appl. Catal., $B, 2015,166,423-431$.

10 H. Li, Z. Xia, J. Chen, L. Lei and J. Xing, Appl. Catal., B, 2015, 168, 105-113.

11 S. K. Choi, S. Kim, J. Ryu, S. K. Lim and H. Park, Photochem. Photobiol. Sci., 2012, 11, 1437-1444.

12 P. Chowdhury, H. Gomaa and A. K. Ray, Chemosphere, 2015, 121, 54-61.
13 A. T. Hodgson, D. Beal and J. E. R. McIlvaine, Indoor Air, 2002, 12, 235-242.

14 Y. Odaka, H. Seto, H. Nakaoka, M. Hanazato, E. Todaka and C. Mori, Indoor Built Environ., 2014, 25, 254-261.

15 I. Sopyan, M. Watanabe, S. Murasawa, K. Hashimoto and A. Fujishima, J. Photochem. Photobiol., A, 1996, 98, 79-86.

16 S. W. Verbruggen, K. Masschaele, E. Moortgat, T. E. Korany, B. Hauchecorne, J. A. Martens and S. Lenaerts, Catal. Sci. Technol., 2012, 2, 2311-2318.

17 T. Nikawa, S.-i. Naya and H. Tada, J. Colloid Interface Sci., 2015, 456, 161-165.

18 I. Jansson, K. Yoshiiri, H. Hori, F. J. García-García, S. Rojas, B. Sánchez, B. Ohtani and S. Suárez, Appl. Catal., A, 2016, 521, 208-219.

19 M.-V. Sofianou, M. Tassi, V. Psycharis, N. Boukos, S. Thanos, T. Vaimakis, J. Yu and C. Trapalis, Appl. Catal., B, 2015, 162, 27-33.

20 D. Meroni, S. Ardizzone, G. Cappelletti, C. Oliva, M. Ceotto, D. Poelman and H. Poelman, Catal. Today, 2011, 161, 169174.

21 S. Wang, X. Zhang, L. Pan, F.-M. Zhao, J.-J. Zou, T. Zhang and L. Wang, Appl. Catal., B, 2015, 164, 234-240.

22 Y. El-Sayed and T. J. Bandosz, Langmuir, 2002, 18, 3213-3218. 23 W. Wei, C. Yu, Q. Zhao, X. Qian, G. Li and Y. Wan, Appl. Catal., B, 2014, 146, 151-161.

24 E. D. Dimotakis, M. P. Cal, J. Economy, M. J. Rood and S. M. Larson, Environ. Sci. Technol., 1995, 29, 1876-1880.

25 S. Kim and S. K. Lim, Appl. Catal., B, 2008, 84, 16-20.

26 S. Kim, M. Kim, Y. K. Kim, S. H. Hwang and S. K. Lim, Appl. Catal., B, 2014, 148, 170-176.

27 X. Chen, L. Liu, P. Y. Yu and S. S. Mao, Science, 2011, 331, 746-750.

28 S. Hoang, S. P. Berglund, N. T. Hahn, A. J. Bard and C. B. Mullins, J. Am. Chem. Soc., 2012, 134, 3659-3662.

29 S. Wang, L. Pan, J.-J. Song, W. Mi, J.-J. Zou, L. Wang and X. Zhang, J. Am. Chem. Soc., 2015, 137, 2975-2983.

30 A. Sinhamahapatra, J.-P. Jeon and J.-S. Yu, Energy Environ. Sci., 2015, 8, 3539-3544.

31 A. Razzaq, A. Sinhamahapatra, T.-H. Kang, C. A. Grimes, J.-S. Yu and S.-I. In, Appl. Catal., B, 2017, 215, 28-35.

32 Y. K. Kim, S. K. Lim, H. Park, M. R. Hoffmann and S. Kim, Appl. Catal., B, 2016, 196, 216-222.

33 S. K. Choi, S. Kim, S. K. Lim and H. Park, J. Phys. Chem. C, 2010, 114, 16475-16480.

34 K. Zhang, Q. Li, L. Zhang, J. Fang, J. Li, F. Qin, Z. Zhang and Y. Lai, Mater. Lett., 2014, 121, 198-201.

35 Y. W. Zhu, S. Murali, W. W. Cai, X. S. Li, J. W. Suk, J. R. Potts and R. S. Ruoff, Adv. Mater., 2010, 22, 3907-3924.

36 A. E. Lewandowska, C. Soutis, L. Savage and S. J. Eichhorn, Compos. Sci. Technol., 2015, 116, 50-57.

37 Y. El-Sayed and T. J. Bandosz, J. Colloid Interface Sci., 2001, 242, 44-51.

38 Y. K. Kim, M. Kim, S. H. Hwang, S. K. Lim, H. Park and S. Kim, Int. J. Hydrogen Energy, 2015, 40, 136-145. 